\title{
Controlling the optical spin Hall effect with light
}

O. Lafont, S. M. H. Luk, P. Lewandowski, N. H. Kwong, P. T. Leung, E. Galopin, A. Lemaitre, J. Tignon, S.

Schumacher, E. Baudin, and R. Binder

Citation: Appl. Phys. Lett. 110, 061108 (2017); doi: 10.1063/1.4975681

View online: http://dx.doi.org/10.1063/1.4975681

View Table of Contents: http://aip.scitation.org/toc/apl/110/6

Published by the American Institute of Physics

\section{Articles you may be interested in}

Electrically driven and electrically tunable quantum light sources

Appl. Phys. Lett. 110, 071102071102 (2017); 10.1063/1.4976197

Comparative determination of Y3Fe5012/Pt interfacial spin mixing conductance by spin-Hall magnetoresistance and spin pumping

Appl. Phys. Lett. 110, 062402062402 (2017); 10.1063/1.4975704

Magnetic field sensor based on coupled photonic crystal nanobeam cavities

Appl. Phys. Lett. 110, 061110061110 (2017); 10.1063/1.4975804

Terahertz plasmonic rectification in a spatially periodic graphene

Appl. Phys. Lett. 110, 061106061106 (2017); 10.1063/1.4975829

Spin-photo-currents generated by femtosecond laser pulses in a ferrimagnetic GdFeCo/Pt bilayer

Appl. Phys. Lett. 110, 072402072402 (2017); 10.1063/1.4976202

Room-temperature vertical-cavity surface-emitting lasers at $4 \mu \mathrm{m}$ with GaSb-based type-Il quantum wells Appl. Phys. Lett. 110, 071104071104 (2017); 10.1063/1.4975813
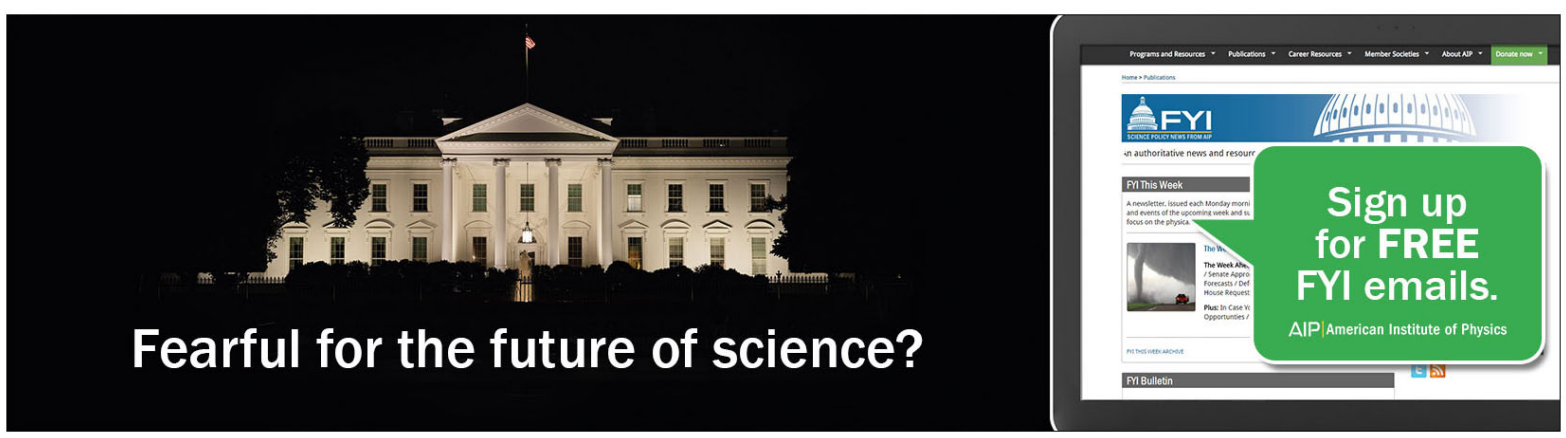


\title{
Controlling the optical spin Hall effect with light
}

\author{
O. Lafont, ${ }^{1}$ S. M. H. Luk, ${ }^{2}$ P. Lewandowski, ${ }^{3}$ N. H. Kwong,${ }^{4}$ P. T. Leung, ${ }^{5}$ E. Galopin, ${ }^{6}$ \\ A. Lemaitre, ${ }^{6}$ J. Tignon, ${ }^{1}$ S. Schumacher, ${ }^{3,4}$ E. Baudin, ${ }^{1}$ and R. Binder ${ }^{2,4}$ \\ ${ }^{1}$ Laboratoire Pierre Aigrain, École Normale Supérieure, PSL Research University, CNRS, \\ Université Pierre et Marie Curie, Sorbonne Universités, Université Paris Diderot, Sorbonne Paris-Cité, \\ 24 rue Lhomond, 75231 Paris Cedex 05, France \\ ${ }^{2}$ Department of Physics, University of Arizona, Tucson, Arizona 85721, USA \\ ${ }^{3}$ Physics Department and Center for Optoelectronics and Photonics Paderborn (CeOPP), \\ Universität Paderborn, Warburger Strasse 100, 33098 Paderborn, Germany \\ ${ }^{4}$ College of Optical Sciences, University of Arizona, Tucson, Arizona 85721, USA \\ ${ }^{5}$ Department of Physics and Institute of Theoretical Physics, The Chinese University of Hong Kong, \\ Hong Kong, China \\ ${ }^{6}$ Laboratoire de Photonique et de Nanostructures, CNRS Route de Nozay, FR-91460 Marcoussis, France
}

(Received 24 December 2016; accepted 20 January 2017; published online 9 February 2017)

\begin{abstract}
The optical spin Hall effect is a transport phenomenon of exciton polaritons in semiconductor microcavities, caused by the polaritonic spin-orbit interaction, which leads to the formation of spin textures. The control of the optical spin Hall effect via light injection in a double microcavity is demonstrated. Angular rotations of the polarization pattern up to $22^{\circ}$ are observed and compared to a simple theoretical model. The device geometry is responsible for the existence of two polariton branches which allows a robust independent control of the polariton spin and hence the polarization state of the emitted light field, a solution technologically relevant for future spin-optronic devices. Published by AIP Publishing. [http://dx.doi.org/10.1063/1.4975681]
\end{abstract}

The detection and manipulation of spins have wide applications in areas ranging from quantum computing, information, and spintronics, ${ }^{1}$ to ubiquitous medical imaging techniques such as Magnetic Resonance Imaging (MRI). Major research efforts have focused on photonic counterparts to magnetic spin systems, including the plasmonic spin Hall effect, ${ }^{2}$ and importantly, wide-ranging investigations of the spin orbit interactions of light. ${ }^{3-7}$ All-optical spin systems combine the benefits of magnetic spin systems and their spin dynamics with the highly developed technology of optical preparation and detection of polarization states (the optical analogue of spin). A promising semiconductor system is a microcavity containing semiconductor quantum wells, where spin states of exciton-polaritons can be created optically and the transverse-electric and transverse-magnetic (TE-TM) splitting yields a spin-orbit interaction that can be described by an effective magnetic field. This, in turn, gives rise to the so-called optical spin Hall effect (OSHE): ${ }^{8-13}$ Since polaritons with different in-plane wave vectors experience different effective magnetic fields, an isotropic distribution of polaritons on a ring in wave vector space can lead to an anisotropic polarization texture or pattern, ${ }^{8,11,14,15}$ both in real (configuration) and wave vector spaces.

In the following, we demonstrate the all-optical manipulation and well-defined control of the polarization/spin texture. Our setup is conceptually simple: a double micocavity provides two polariton branches, ${ }^{16}$ one that contains the offaxis $(\mathbf{k} \neq 0)$ polaritons, which actually form the OSHE pattern and which do not couple directly to the external field, and one that contains the $\mathbf{k}=0$ polaritons that are directly pumped by an external field at normal incidence. It allows for robust steady state control that would be required in future device applications. We show that a circularly polarized pump rotates the far-field polarization/spin texture. The rotation angle generally increases with the pump spot size, and for Gaussian beam profiles, its dependence on the pump power approaches a simple arctangent law in the limit of large spot sizes. ${ }^{17}$

The sample consists of two coupled $\lambda / 2 \mathrm{Ga}_{0.05} \mathrm{Al}_{0.95} \mathrm{As}$ cavities embedded between three $\mathrm{Ga}_{0.05} \mathrm{Al}_{0.95} \mathrm{As} / \mathrm{Ga}_{0.8} \mathrm{Al}_{0.2} \mathrm{As}$ Bragg mirrors with 25 (back), 17.5 (middle), and 17.5 (front) pairs, respectively (Fig. 1(a)). The nominal Q factor is around $10^{5}$ and the middle Bragg mirror induces an approximately $10 \mathrm{meV}$ coupling between the bare cavity modes. In each cavity, 3 sets of four $7 \mathrm{~nm} \mathrm{GaAs}$ quantum wells are inserted at the antinodes of the field resulting in a $13 \mathrm{meV}$ Rabi splitting. A wedge is introduced in the cavities thickness during the growth by molecular beam epitaxy. This wedge allows to tune the cavity mode energies with respect to the bare excitonic mode energy. Experiments are performed at $T=6 \mathrm{~K}$, the excitation laser is provided by a single mode Ti:Sapphire laser, Fig. 1(b). The sample is excited at normal incidence with circularly polarized light. The pump beam is hidden by a spatial filter. The polarization-resolved far-field spectroscopy is obtained by projecting the Fourier plane of the detection objective on a cooled CCD camera.

Figure 2(a) shows a typical far-field pattern of the elastically scattered light for the degree of linear polarization given by the Stokes parameter $S_{1}(\mathbf{k})$, where $\mathbf{k}$ is the polariton's in-plane wavevector, which determines the direction in which the emitted field propagates in the far field. The Stokes parameter $S_{1}$ is positive (negative) if the polarization state of the field is mostly $\mathrm{x}(\mathrm{y})$. The polarization pattern is centro-symmetric and is composed of four quadrants which are mainly linearly polarized. Along the horizontal and vertical axes, i.e., for momentum along the analyzer direction, a clear splitting in momentum space between the horizontal 

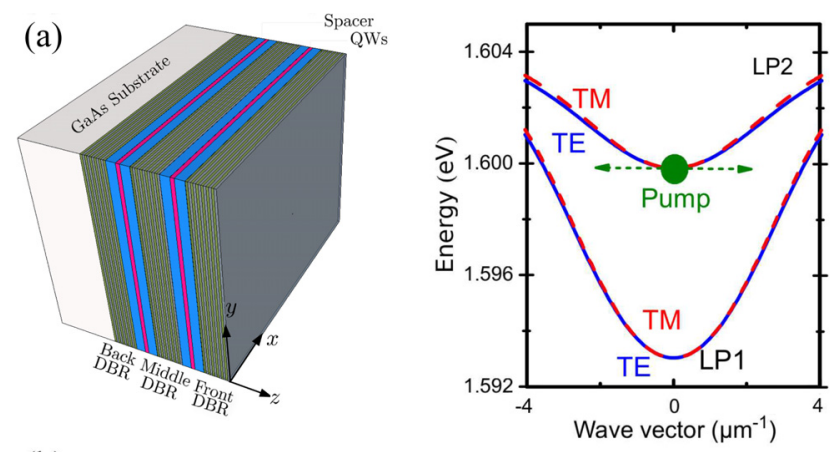

(b) Fourier plane Powermeter

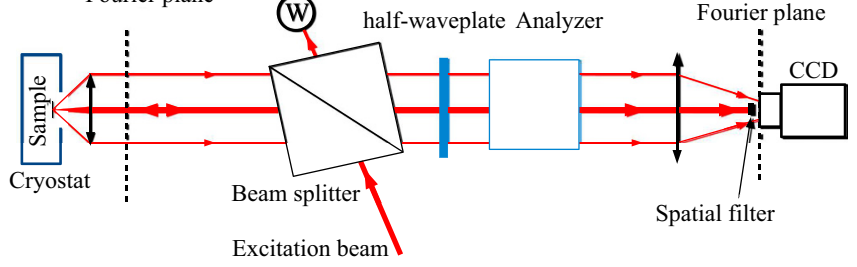

FIG. 1. (a) Sketch of the double microcavity and the corresponding dispersion relationship of the two lower polariton branches. (b) Sketch of the experimental setup.

(a)

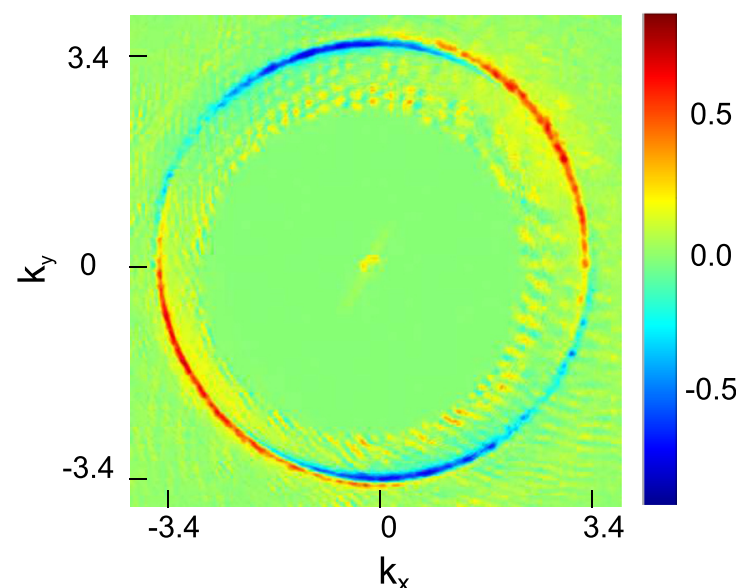

(b)

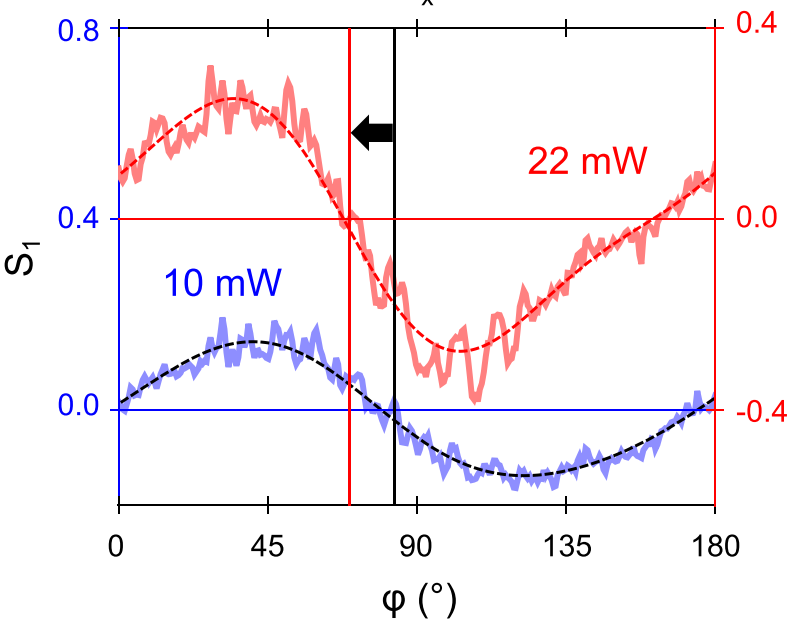

FIG. 2. (a) Experimentally observed far-field polarization texture $S_{1}(\mathbf{k})$, obtained for a $22 \mathrm{~mW}$ "+" circularly polarized pump incident on the cavity. (b) Experimental angular variation of the radial average, $\bar{S}_{1}^{(n)}$, for two incident excitation powers (blue $10 \mathrm{~mW}$ and red $22 \mathrm{~mW}$ ) displaying a lightinduced shift of $-13.8^{\circ} \pm 0.7^{\circ}$ of the angular orientation as indicated by the positions of the zero crossings (vertical lines). The dashed lines correspond to the fit described in the text. and vertical branches is observed. This momentum splitting results from the large TE-TM splitting of the lower polaritonic branches at such large momentum.

The pattern shown in Fig. 2(a) can be manipulated in a well-controlled fashion by the application of a pump field. Before discussing the experimental results of the control effect, we introduce some useful theoretical concepts. A simple pseudo-spin model of the optical spin Hall effect ${ }^{9,11}$ allows to gain insight into the formation and control of the pseudospin pattern. The pseudo-spin vector $\mathbf{S}$ is given in terms of the Stokes parameters formed by the polariton wave functions. The evolution of $\mathbf{S}$ is given by the following torque equation:

$$
\dot{\mathbf{S}}(\mathbf{k})=\mathbf{B}(\mathbf{k}) \times \mathbf{S}(\mathbf{k})-\gamma \mathbf{S}(\mathbf{k})+\mathbf{R}(\mathbf{k})
$$

which includes the effective magnetic field $\mathbf{B}(\mathbf{k})$, a decay term (with decay rate $\gamma$ ), and a source $\mathbf{R}$. Positive (negative) $S_{1}$ corresponds to dominant x (y) linear polarization components of the polariton fields, and positive (negative) $S_{3}$ to "+" (“-") circular polarizations. Throughout this paper, we assume a "+" circularly polarized pump, and hence $\mathbf{R}=(0,0, R)$. In steady state, we find $S_{1}=A\left(\gamma B_{2}+B_{1} B_{3}\right)$ with $A=(R / \gamma)\left(\mathbf{B}^{2}+\gamma^{2}\right)^{-1}$. In the low density limit, the magnetic field is restricted to the 1-2 plane of the threedimensional pseudo-spin space $\left(B_{3}=0\right)$, and has the angle dependence $\mathbf{B}(\mathbf{k})=\Delta_{k}(\cos (2 \phi), \sin (2 \phi), 0)$, where $\Delta_{k}$ is the splitting between the TE and TM modes, and $\phi$ is the azimuthal angle of the vector $\mathbf{k} .^{8,11}$ For example, for $\phi=$ $45^{\circ}$ the torque of the B-field has only a positive $B_{2}$ component and rotates $\mathbf{S}$ towards positive $S_{1}$, i.e., toward $\mathrm{x}$ polarization until it settles to steady state (in this case in the 1-3 plane). At $\phi=-45^{\circ}$, the negative $B_{2}$ component of $\mathbf{B}$ would favor y-polarization. Indeed, for $B_{3}=0$ we have $S_{1}=A \gamma B_{2}(\phi)$, creating the familiar ${ }^{9,11}$ polarization map of $S_{1}$ with the polariton in-plane momentum polar angle $\phi$.

To gain a quantitative insight in the OSHE, it is necessary to determine the orientation of the polarization farfield pattern. This is made possible by integrating the intensity observed in both linear polarization channels on the elastic circle over momentum amplitude (in the range of 3.16-3.64 $\mu \mathrm{m}^{-1}$ ). This yields a radial average of the degree of linear polarization given by $\bar{S}_{1}^{(n)}=\frac{X-Y}{X+Y}$ as represented in Fig. 2(b), where $X$ and $Y$ are the integrated intensities in horizontal and vertical polarizations, respectively, and the superscript " $n$ " denotes a normalized Stokes parameter (i.e., divided by the total power $\mathrm{X}+\mathrm{Y})$. The radial average $\bar{S}_{1}^{(n)}$ shows a predominant $\sin \left(2\left(\phi-\phi_{0}\right)\right)$ dependence, as shown in Fig. 2(b), for two different powers. The observed peakto-peak linear polarization amplitude is the characteristic of our whole measurement datasets regardless of power. We denote by $\phi_{0}$ the angle at which $\bar{S}_{1}(\phi)$ vanishes. The rotation angle $\phi_{0}$ is a quantitative measure of the OSHE control. When the power of the incoming pump beam is changed, we observe a shift of the orientation $\phi_{0}$ of the average degree of linear polarization while the far-field polarization pattern 2(a) is almost unchanged.

The $\bar{S}_{1}^{(n)}$ data are fitted using a multipolar expansion up to the eighth order: $\bar{S}_{1}^{(n)}=A_{d} \sin \left(\phi+\theta_{D}\right)+A_{q} \sin (2(\phi$ $\left.\left.-\phi_{0}\right)\right)+A_{o} \sin \left(4 \phi+\theta_{0}\right)$ where sinusoidal terms are 
dipolar, quadrupolar, and octopolar terms in a consecutive order. According to the theoretical model developed here, the circularly polarized excitation-linearly polarized detection scheme has the key advantage over the polarization configuration originally considered for the OSHE observation ${ }^{11}$ that when the pump power is changed, only the quadrupolar phase is modified. In this configuration, the various sources of experimental imperfections (excitation and detection polarization miscalibration, built-in stress, optical misalignment, $k=0$ position on image) do not affect the quadrupolar phase except for spherical aberrations of the imaging setup and polarization-dependent elastic scatterings, which are intensity-independent.

In Fig. 3(a), we plot the zero crossing $\phi_{0}$ extracted from data analysis as a function of power. The standard errors obtained from fitting are multiplied by a factor $\sqrt{\beta_{c} / \beta_{d}}$, where $\beta_{c}=3.7^{\circ}$ is the characteristic autocorrelation angle of residuals, and $\beta_{d}=0.6^{\circ}$ is the discretization step angle of data to account for the spatial correlation effects on fitting results. The $68 \%$ of expanded uncertainty indicated by the error bars in Fig. 3(a) takes into account the experimental accuracy of $0.6^{\circ}$ due to the waveplate positioning. The experimental data in Fig. 3(a) exhibit a smooth orientational shift of the OSHE with the increasing power reaching up to $22^{\circ}$. We

(a)

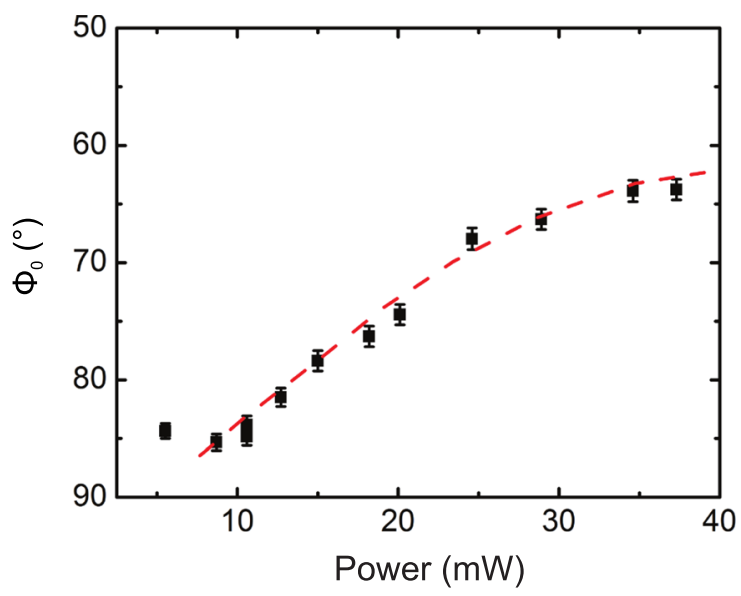

(b)

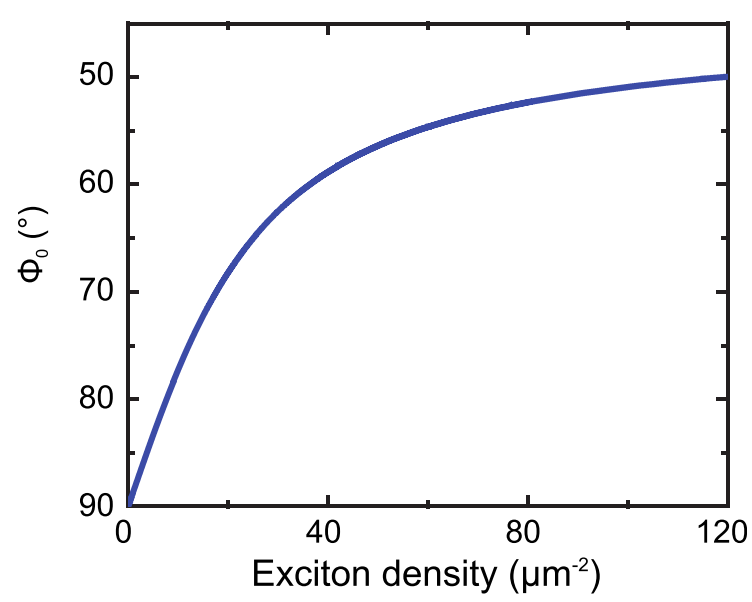

FIG. 3. (a) Experimental angular orientation (zero-crossings) $\phi_{0}$ vs. incident excitation power. The thin dashed curve is merely a guide for the eye. (b) Theoretical angular orientation (zero-crossings) $\phi_{0}$ of the radial average, $\bar{S}_{1}^{(n)}$, from Eq. (2). The horizontal axis represents the exciton density, which is a fraction of the LP2 polariton density. The power range in (b) is estimated to correspond to the range of exciton densities in (a), see Ref. 17. have verified that this response is independent of the choice of the analyzer orientation and that reversing the circular polarization of the pump reverses the polarization orientational shift. We have not observed a bistable behaviour.

Our results indicate that using a circularly polarized optical pump allows to create substantial densities of circularly polarized polaritons. As a result of Coulombic polariton interactions, the excited polaritons create a $B_{3}$ component of the effective magnetic field. This has been found in Refs. 18-20 for the case of a single cavity where the $B_{3}$ component has been reported to result in a self-induced Larmor precession. In Ref. 17, we give a generalization of the interactions to the case of a double cavity which is technologically relevant as the two lower polariton branches can be functionalized as control (here the pump) and signal channel (here the elastic circle). For the present simplified pseudo-spin argument, it suffices to assume $B_{3} \neq 0$ as a consequence of the optical pumping. Then the nutation of an initially created pseudo-spin along the $S_{3}$-direction is more complex. Now the steady state solution is

$$
S_{1}=A\left(\gamma B_{2}+B_{1} B_{3}\right) \propto \cos (2 \phi+\Theta),
$$

where $\tan \Theta=-\gamma / B_{3}$. This shows that increasing $B_{3}$, i.e., increasing the optical pumping, rotates the stationary pseudospin texture by the angle $\Theta$.

Theoretical predictions from the pseudo-spin model are shown in Fig. 3(b) in the relevant power range for a direct comparison to Fig. 3(a). The simplified pseudo-spin model slightly overestimates the rotation angle due to the infinite spot size assumption. The experimental pump beam is a Gaussian beam with a full-width half maximum of approximately $50 \mu \mathrm{m}$. The corresponding exciton density is obtained by writing the energy conservation of the cavity, balancing incident, reflected and transmitted powers with a loss through exciton recombination $P_{\text {inc }} \sim \gamma_{\text {rec }}|p|^{2}$, where $P_{\text {inc }}$ is the incident power, $|p|^{2}$ is the exciton density in each quantum well (approximately the same for all quantum wells), $\gamma_{\text {rec }}$ is the recombination rate (including radiative and non-radiative recombination), and the proportionality constant (omitted here) is given in Ref. 17. Considering a lifetime $\tau_{\text {rec }}=\hbar / \gamma_{\text {rec }}$ of about $100 \mathrm{ps}$ as expected for our experiment, the scales of Figs. 3(a) and 3(b) are in direct correspondence. Consequently, the simplified pseudo-spin model gives a correct order of magnitude for the amplitude and saturation of the control of the OSHE.

In conclusion, the clear all-optical control on the pseudo-spin currents obtained using a simple double microcavity in the elastic scattering regime allows to envision the logic spin-optronic network ${ }^{21-23}$ based on the polaritonic devices. Previous studies on the control of the OSHE were done in the Bose-Einstein condensation regime where polaritonic Bose-Einstein condensate dynamics is entangled with the pseudo-spin dynamics. ${ }^{8}$ This work shows that a simple and independent control is possible provided that two independent polaritonic channels are used. Future developments for spinoptronic-based computation ${ }^{24}$ involve the lateral confinement of polaritons by patterning the microcavity ${ }^{25-29}$ and the temporal control of the input data by using short pulse sequences. 
We gratefully acknowledge the financial support from the U.S. NSF under Grant No. ECCS-1406673, TRIF SEOS, and the German DFG (TRR142, SCHU 1980/5, Heisenberg program). We thank Kyle Gag for helpful discussions.

${ }^{1}$ D. D. Awschalom, D. Loss, and N. Samarth, Semiconductor Spintronics and Quantum Computation (Springer, Berlin, 2002).

${ }^{2}$ Sh. Xiao, F. Zhong, H. Liu, Sh. Zhu, and J. Li, Nat. Commun. 6, 8360 (2015).

${ }^{3}$ K. Y. Bliokh, F. J. Rodriguez-Fortuno, F. Nori, and A. V. Zayats, Nat. Photonics 9, 796 (2015).

${ }^{4}$ E. Brasselet, N. Murazawa, H. Misawa, and S. Juodkazis, Phys. Rev. Lett. 103, 103903 (2009).

${ }^{5}$ V. S. Liberman and B. Ya. Zeldovich, Phys. Rev. B 46, 5199 (1992).

${ }^{6}$ O. G. Rodriguez-Herrera, D. Lara, K. Y. Bliokh, E. A. Ostrovskaya, and C. Dainty, Phys. Rev. Lett. 104, 253601 (2010).

${ }^{7}$ X. Yin, Z. Ye, J. Rho, Y. Wang, and X. Zhang, Science 339, 1405 (2013).

${ }^{8}$ E. Kammann, T. C. H. Liew, H. Ohadi, P. Cilibrizzi, P. Tsotsis, Z. Hatzopoulos, P. G. Savvidis, A. V. Kavokin, and P. G. Lagoudakis, Phys. Rev. Lett. 109, 036404 (2012).

${ }^{9}$ A. Kavokin, G. Malpuech, and M. Glazov, Phys. Rev. Lett. 95, 136601 (2005).

${ }^{10}$ W. Langbein, I. Shelykh, D. Solnyshkov, G. Malpuech, Y. G. Rubo, and A. Kavokin, Phys. Rev. B 75, 075323 (2007).

${ }^{11}$ C. Leyder, M. Romanelli, J. Ph. Karr, E. Giacobino, T. C. H. Liew, M. M. Glazov, A. V. Kavokin, G. Malpuech, and A. Bramati, Nat. Phys. 3, 628 (2007).

${ }^{12}$ F. Manni, K. G. Lagoudakis, T. K. Paraiso, R. Cerna, Y. Leger, T. C. H. Liew, I. A. Shelykh, and A. V. Kavokin, Phys. Rev. B 83, 241307 (2011).

${ }^{13}$ M. Maragkou, C. E. Richards, T. Ostatnicky, A. J. D. Grundy, J. Zajac, M. Hugues, W. Langbein, and P. G. Lagoudakis, Opt. Lett. 36, 1095 (2011).

${ }^{14}$ A. H. Hielscher, A. A. Eick, J. R. Mourant, D. Shen, J. P. Freyer, and I. J. Bigio, Opt. Express 1, 441 (1997).

${ }^{15}$ C. Schwartz and A. Dogariu, J. Opt. Soc. Am. A 25, 431 (2008).
${ }^{16}$ V. Ardizzone, P. Lewandowski, Y. C. Tse, N. H. Kwong, M. H. Luk, A. Lücke, M. Abbarchi, E. Baudin, E. Galopin, J. Bloch, A. Lemaitre, P. T. Leung, P. Roussignol, R. Binder, J. Tignon, and S. Schumacher, Sci. Rep. 3, 3016 (2013).

${ }^{17}$ M. H. Luk, P. Lewandowski, N. H. Kwong, M. Babilon, O. Lafont, K. P. Chan, P. T. Leung, J. Tignon, S. Schumacher, E. Baudin, and R. Binder (to be published).

${ }^{18}$ D. N. Krizhanovskii, D. Sanvitto, I. A. Shelykh, M. M. Glazov, G. Malpuech, D. D. Solnyshkov, A. V. Kavokin, S. Ceccarelli, M. S. Skolnick, and J. S. Roberts, Phys. Rev. B 73, 73303 (2006).

${ }^{19}$ P. G. Lagoudakis, P. G. Savvidis, J. J. Baumberg, D. M. Whittaker, P. R. Eastham, M. S. Skolnick, and J. S. Roberts, Phys. Rev. B 65, 161310 (2002).

${ }^{20}$ I. A. Shelykh, A. V. Kavokin, Y. G. Rubo, T. C. H. Liew, and G. Malpuech, Semicond. Sci. Technol. 25, 013001 (2010).

${ }^{21}$ H. Flayac, D. D. Solnyshkov, G. Malpuech, and I. A. Shelykh, Phys. Rev. B 87, 075316 (2013).

${ }^{22}$ T. Gao, C. Antón, T. C. H. Liew, M. D. Martín, Z. Hatzopoulos, L. Viña, P. S. Eldridge, and P. G. Savvidis, Appl. Phys. Lett. 107, 011106 (2015).

${ }^{23}$ T. C. H. Liew, Y. G. Rubo, and A. V. Kavokin, Phys. Rev. Lett. 101, 187401 (2008).

${ }^{24}$ D. D. Solnyshkov, O. Bleu, and G. Malpuech, Superlattices Microstruct. 83, 466 (2015).

${ }^{25}$ C. Antón, T. C. H. Liew, G. Tosi, M. D. Martín, T. Gao, Z. Hatzopoulos, P. S. Eldridge, P. G. Savvidis, and L. Viña, Appl. Phys. Lett. 101, 261116 (2012).

${ }^{26}$ O. El Daïf, A. Baas, T. Guillet, J.-P. Brantut, R. Idrissi Kaitouni, J. L. Staehli, F. Morier-Genoud, and B. Deveaud, Appl. Phys. Lett. 88, 061105 (2006).

${ }^{27}$ H. Flayac and I. G. Savenko, Appl. Phys. Lett. 103, 201105 (2013).

${ }^{28}$ F. Marsault, H. S. Nguyen, D. Tanese, A. Lemaître, E. Galopin, I. Sagnes, A. Amo, and J. Bloch, Appl. Phys. Lett. 107, 201115 (2015).

${ }^{29}$ A. V. Nalitov, D. D. Solnyshkov, and G. Malpuech, Phys. Rev. Lett. 114, 116401 (2015). 